$$
\left\{\pi(x)-\pi\left(x^{1 / 2}\right)\right\} \log x^{1 / 2} \leqq \theta(x) \leqq \pi(x) \log x,
$$

Tschebyschef's theorem

$$
\alpha \frac{x}{\log x} \leqq \pi(x) \leqq \gamma \frac{x}{\log x}
$$

now follows at once.

\title{
REFERENCE
}

1. E. Landau, Handbuch der Lehre von der Verteilung der Primzahlen, vol. 1.

NEW YoRK UNIVERSITY

\section{THE RADICAL OF A NON-ASSOCIATIVE RING}

W. E. JENNER

In this paper a definition is proposed for the radical of a non-associative ring. Our results are somewhat similar to those given for algebras by Albert in [3], ${ }^{1}$ but the difficulties that arose in the earlier theory from absolute divisors of zero have been overcome. With slight modifications, the present proofs are applicable to algebras.

A non-associative ring $\Re$ is an additive abelian group closed under a product operation with respect to which the two distributive laws hold. Multiplication on the right (left) by a fixed element $x \in \Re$ determines an endomorphism $R_{x}\left(L_{x}\right)$ of $\Re$ as an additive group. For $x, y \in \Re$,

$$
x \cdot y=x R_{y}=y L_{x} .
$$

The $R_{x}$ and $L_{y}$ generate an associative ring $\mathfrak{A}$ called the transformation ring of $\Re$. Clearly $\Re$ can be construed as a representation space for $\mathfrak{A}$, and this representation is faithful. The two-sided ideals of $\mathfrak{R}$, which are defined as for associative rings, are exactly the $\mathscr{\Re}$-subspaces of $\Re$. The theory of ring homomorphisms goes over intact to the non-associative case.

A nonzero element $a \in \Re$ is called an absolute divisor of zero if $a \cdot x=x \cdot a=0$ for all $x \in \mathfrak{R}$. If $\mathfrak{A}$ has a unit element $I$ and $\mathfrak{R}$ contains no absolute divisors of zero, then the unit element $I$ is the identity

Received by the editors March 19, 1949.

${ }^{1}$ Numbers in brackets refer to the references cited at the end of the paper. 
mapping on $\Re$. For if $a I=b$ where $a, b \in \Re$, then $a I-b=0=(a I-b) I$ $=(a-b) I,(a-b) \mathfrak{A}=0$ and so $a=b$.

We now assume that the minimum condition holds in $\mathscr{P}$. It has been shown in [5, Theorems 3 and 14] that for the case of simple rings this is equivalent to the assumption that $\Re$ can be regarded as an algebra of finite dimension over a certain field.

TheOREM 1. Let $\mathfrak{A}$ satisfy the minimum condition and $\Re$ contain no absolute divisors of zero. Then $\Re=\Re_{1} \oplus \Re_{2} \oplus \cdots \oplus \Re_{n}$, where the $\Re_{i}$ are simple if and only if $\mathfrak{A}=\mathfrak{A}_{1} \oplus \mathfrak{A}_{2} \oplus \cdots \oplus \mathfrak{A}_{n}$ where the $\mathfrak{A}_{i}$ are simple, and conversely.

Proof. If $\Re=\Re_{1} \oplus \Re_{2} \oplus \cdots \oplus \Re_{n}$ where the $\Re_{i}$ are simple, then $\mathfrak{A}=\mathfrak{A}_{1} \oplus \mathfrak{A}_{2} \oplus \cdots \oplus \mathfrak{A}_{n}$ where $\mathfrak{A}_{i}$ is the transformation ring for $\mathfrak{R}_{i}$. Since $\mathfrak{R}_{i}$ is an irreducible $\mathfrak{A}_{i}$-space, it follows that $\mathfrak{A}_{i}$ is simple. Conversely, suppose $\mathfrak{A}=\mathfrak{A}_{1} \oplus \mathfrak{A}_{2} \oplus \cdots \oplus \mathfrak{A}_{n}$ where the $\mathfrak{A}_{i}$ are simple rings generated by pairwise orthogonal idempotents $E_{i}$ in the center of $\mathfrak{A}$. Clearly $\Re=\Re_{1} \oplus \Re_{2} \oplus \cdots \oplus \Re_{n}$, where $\Re_{i}=\Re E_{i}$. It is easily seen that $\mathfrak{A}_{i}$ is the transformation ring for $\Re_{i}$. If $\Re_{i}$ has a proper ideal $\mathfrak{M}$, then $\mathfrak{R}_{i}=\mathfrak{M} \oplus \mathfrak{N}$, a direct sum of $\mathfrak{A}_{i}$-subspaces since $\mathfrak{A}_{i}$ is semisimple. Let $\mathfrak{S}$ be the ideal of $\mathfrak{A}_{i}$ which annihilates $\mathfrak{M}$. For every nonzero element $x$ in $\mathfrak{N}, R_{x}$ and $L_{x}$, at least one of which must be nonzero, are in $\subseteq$. Clearly $E_{i}$ is not in $\subseteq$ and so $\subseteq$ is a proper ideal of $\mathfrak{A}_{i}$, which is impossible. Therefore $\mathfrak{R}_{i}$ is simple.

CoRollary. Under the assumptions of Theorem $1, \Re$ is simple if and only if $\mathfrak{A}$ is simple.

The ring $\Re$ is defined to be semisimple if the following conditions are satisfied:

(i) $\mathscr{A}$ satisfies the minimum condition.

(ii) $\Re$ contains no absolute divisors of zero.

(iii) $\Re$ is a direct sum of simple rings.

We now eliminate the restriction that $\Re$ contain no absolute divisors of zero. Suppose $\Re$ has an ideal $\mathfrak{W}$. Let $\mathfrak{S}$ be the set consisting

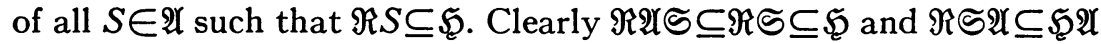
$\subseteq \mathfrak{W}$. Furthermore, $\mathfrak{S}$ is an additive subgroup and so an ideal of $\mathfrak{A}$. Let $[x]$ and $[y]$ be any two residue classes of $\Re-\mathfrak{W}$. Then $[x] \cdot[y]$ $=[x \cdot y]=\left[x R_{y}\right]=\left[y L_{x}\right]$. If $[x U]=[x V]$ for all $[x] \in \Re-\mathfrak{S}$ where $U, V \in \mathfrak{A}$, then $U-V \in \mathfrak{S}$ and so right (left) multiplication by a fixed element of $\Re-\mathfrak{S}$ determines a unique residue class of $\mathfrak{A}-\mathfrak{S}$. Then it is easy to prove that $\mathfrak{A}-\mathfrak{S}$ is isomorphic to the transformation ring for $\mathfrak{R}-\mathfrak{S}$. It follows immediately that if $\mathfrak{A}$ satisfies the minimum 
condition, then so does the transformation ring for any difference ring of $\Re$.

Let $\mathfrak{M}_{1}$ be the ideal consisting of zero and all absolute divisors of zero in $\Re$. Continuing by induction, let $\mathfrak{M}_{i+1}$ be the set consisting of all $x \in \Re$ such that $a \cdot x$ and $x \cdot a \in \mathfrak{M}_{i}$ for all $a \in \Re$. The $\mathfrak{M}_{i}$ are ideals of $\Re$ and $\mathfrak{M}_{i} \subseteq \mathfrak{M}_{i+1}{ }^{2}$

Lemma 1. $\mathfrak{M}_{j+1}$ is the set consisting of all $x \in \Re$ such that $R_{x}$ and $L_{x}$ are left-annihilators of $\mathfrak{A}^{j}$.

Proof. Suppose $x \in \mathfrak{M}_{j+1}$. Then $a R_{x}$ and $a L_{x} \in \mathfrak{M}_{j}$ for all $a \in \Re$. Each succeeding application of transformation in $\mathscr{A}$ gives rise to an element in the preceding $\mathfrak{M}_{i}$. Clearly $a R_{x} T_{1} T_{2} \ldots T_{j}=a L_{x} T_{1} T_{2} \ldots$ $T_{j}=0$ for all $a \in \Re$, where $T_{1}, T_{2}, \cdots, T_{j}$ are arbitrary elements of श. Therefore $R_{x} T_{1} T_{2} \cdots T_{j}=L_{x} T_{1} T_{2} \cdots T_{j}=0$ and so $R_{x}$ and $L_{x}$ are left annihilators of $\mathfrak{A}^{j}$. Conversely, if $a R_{x} T_{1} T_{2} \cdots T_{j}$ $=a L_{x} T_{1} T_{2} \ldots T_{j}=0$ for all $a \in \Re$, where $T_{1}, T_{2}, \cdots, T_{j}$ are arbitrary elements of $\mathfrak{A}$, then $a R_{x} T_{1} T_{2} \ldots T_{j-1}$ and $a L_{x} T_{1} T_{2} \ldots T_{j-1}$ are in $\mathfrak{M}_{1}$ by definition of $\mathfrak{M}_{1}$. Continuing in this way, it is easily seen that $a R_{x}$ and $a L_{x}$, that is, $a \cdot x$ and $x \cdot a$ are in $\mathfrak{M}_{j}$ for all $a \in \Re$ and so $x \in \mathfrak{M}_{j+1}$.

Lemma 2. If $\mathfrak{A}$ satisfies the minimum condition, then there exists a least integer $l$ such that $\mathfrak{M}_{l}=\mathfrak{M}_{l+1}$.

PROof. Let $k$ be the first integer for which $\mathfrak{A}^{k-1}=\mathfrak{A}^{k}$. The existence of $k$ is insured by the minimum condition on $\mathfrak{A}$. Suppose $x$ is an element of $\mathfrak{M}_{k+1}$ not in $\mathfrak{M}_{k}$. By Lemma $1, R_{x}$ and $L_{x}$ would be leftannihilators of $\mathfrak{\Re}^{k}$ but not both of $\mathfrak{Y}^{\mathfrak{k}-1}$. This is impossible, and so $\mathfrak{M}_{k+1}=\mathfrak{M}_{k}$. Therefore $k$ is an upper bound for $l$ and the lemma is proved.

In particular, if $\mathfrak{A}$ has a unit element, then $\mathfrak{M}_{2}=\mathfrak{M}_{1}$, since $\mathfrak{A}$ can have no left-annihilators.

Theorem 2. Let $\mathfrak{A}$ satisfy the minimum condition. Then $\mathfrak{R}-\mathfrak{M}_{l}$ has no absolute divisors of zero. Furthermore, $\mathfrak{M}_{l}$ is contained in every ideal $\mathfrak{S}$ for which $\mathfrak{R}-\mathfrak{S}$ has no absolute divisors of zero.

PRoof. If $a \cdot x$ and $x \cdot a \in \mathfrak{M}_{l}$ for all $a \in \mathfrak{R}$, then $x \in \mathfrak{M}_{l+1}=\mathfrak{M}_{l}$. This proves the first part. Suppose $\mathfrak{R}-\mathfrak{S}$ has no absolute divisors of zero. Clearly $\mathfrak{M}_{1} \subseteq \mathfrak{W}$. Now assume $\mathfrak{M}_{i} \subseteq \mathfrak{W}$. If $x \in \mathfrak{M}_{i+1}$, then both $a \cdot x$ and $x \cdot a \in \mathfrak{M}_{i} \subseteq \mathfrak{S}$ for all $a \in \mathfrak{R}$ and so $x \in \mathfrak{S}$. Therefore $\mathfrak{M}_{2} \subseteq \mathfrak{S}$.

Lemma 3. If $\mathfrak{A}$ satisfies the minimum condition and $\mathfrak{R}-\mathfrak{S}$ is semi-

\footnotetext{
${ }^{2}$ In the case of Lie rings, the $\mathfrak{M}_{i}$ constitute the upper central chain.
} 
simple, then $\mathfrak{R} \subseteq \mathfrak{S}$ where $\mathfrak{N}$ is the radical of $\mathfrak{A}$.

PRoof. The transformation ring for $\mathfrak{R}-\mathfrak{S}$ is isomorphic to $\mathfrak{A}-\mathfrak{S}$ where $\mathfrak{S}$ is the ideal consisting of all $S \in \mathfrak{A}$ such that $\mathfrak{R} S \subseteq \mathfrak{G}$. By Theorem $1, \mathfrak{A}-\mathfrak{S}$ is semisimple. Therefore $\mathfrak{R} \subseteq \mathfrak{S}$, and so $\mathfrak{R} \subseteq \mathfrak{R S}$ $\subseteq \mathfrak{W}$.

We now consider the ideal $\mathfrak{M}_{l}$ for the ring $\mathfrak{R}-\mathfrak{R} \Re$. The existence of this ideal follows from Lemma 2 since the transformation ring for $\Re-\Re \mathfrak{R}$ satisfies the minimum condition. Let $\mathfrak{M}$ be the complete reciprocal image of $\mathfrak{M}_{l}$ under the natural homomorphism $\Re \rightarrow \Re$ $-\mathfrak{R}$. The ideal $\mathfrak{M}$ will be call $\mathrm{d}$ the radical of $\mathfrak{R}$. Since $\mathfrak{R}-\mathfrak{M}$ $\cong(\mathfrak{R}-\mathfrak{R} \mathfrak{N})-\mathfrak{M}_{l}$, it follows from Theorem 2 that $\Re-\mathfrak{M}$ has no absolute divisors of zero. The transformation ring for $\Re-\mathfrak{M}$ is isomorphic to $\mathfrak{U}-\mathfrak{S}$, where $\mathfrak{S}$ is the ideal consisting of all $S \in \mathfrak{A}$ such that $\mathfrak{R S \subseteq M}$. Clearly $\mathfrak{R} \subseteq \subseteq$ and so $\mathfrak{A}-\subseteq$ is semisimple. Theorem 1 then implies

Theorem 3. Let $\mathfrak{A}$ satisfy the minimum condition. Then $\mathfrak{R}-\mathfrak{M}$ is semisimple.

That $\mathfrak{M}$ is the minimal ideal having this property is shown by

THEOREM 4. If $\mathfrak{A}$ satisfies the minimum condition and $\mathfrak{R}-\mathfrak{S}$ is semisimple for some ideal $\mathfrak{E}$, then $\mathfrak{M} \subseteq \mathfrak{S}$.

Proof. By Lemma 3, $\mathfrak{R} \subseteq \mathfrak{S}$. Clearly $(\Re-\mathfrak{R})-(\mathfrak{Y}-\mathfrak{R} \mathfrak{R}) \cong \mathfrak{R}$ $-\mathfrak{S}$ and so by Theorem $2, \mathfrak{M}_{l} \subseteq \mathfrak{S}-\mathfrak{R} \Re$, which implies $\mathfrak{M} \subseteq \mathfrak{G}$.

The writer is indebted to D. C. Murdoch for suggesting Lemma 1.

\section{REFERENCES}

1. A. A. Albert, Non-associative algebras I. Fundamental concepts and isotopy, Ann. of Math. (2) vol. 43 (1942) pp. 685-707.

2. - Non-associative algebras II. New simple algebras, Ann. of Math. (2) vol. 43 (1942) pp. 708-723.

3. - The radical of a non-associative algebra, Bull. Amer. Math. Soc. vol. 48 (1942) pp. 891-897.

4. N. Jacobson, $A$ note on non-associative algebras, Duke Math. J. vol. 3 (1937) pp. 544-548.

5. - Structure theory of simple rings without finiteness assumptions, Trans. Amer. Math. Soc. vol. 57 (1945) pp. 228-245.

The University of British Columbia 